'Departamento de Laboratorios Clínicos, Facultad de Medicina, Pontificia Universidad Católica de Chile. Santiago, Chile.

2Departamento de Nutrición, Diabetes y Metabolismo, Facultad de Medicina, Pontificia Universidad Católica de Chile. Santiago, Chile.

${ }^{3}$ Programa Magíster en

Bioestadística, Facultad de Medicina, Universidad de Chile. Santiago, Chile. ${ }^{a}$ Residente de Laboratorio Clínico. besidente de Nutrición Clínica y Diabetología. 'Bioquímico, Magíster en Bioestadística.

*Francisco Vera y Paulina Jofré comparten primera autoría del artículo.

Trabajo no recibió financiamiento. Los autores declaran no tener conflictos de interés.

Recibido el 14 de mayo de 2020, aceptado el 13 de enero de

Correspondencia a:

Dr. David Rodríguez Departamento de Laboratorios Clínicos, Facultad de Medicina, Pontificia Universidad Católica de Chile. drodriguez11@uc.cl

\section{Marcadores inmunológicos de diabetes tipo 1: análisis de 4.164 perfiles en un centro de salud de Chile}

\author{
FRANCISCO VERA ${ }^{1, \mathrm{a}}$, PAULINA JOFRÉ ${ }^{2, \mathrm{~b}}$, \\ DAVID RODRÍGUEZ ${ }^{1, a}$, RODRIGO LAGOS ${ }^{3, c}$, \\ TERESITA QUIROGA ${ }^{1}$, BRUNO GRASSI ${ }^{2}$, FELIPE POLLAK ${ }^{2}$
}

\section{Analysis of 4,164 type 1 diabetes antibody profiles in a Chilean health centre}

Background: Previous studies have assessed the role of Type 1 diabetes (DM1) antibodies as predictors of the natural history of disease. Aim: To determine the frequency and combinations of positivity for DM1 antibodies in patients with DM1 and the relationship between antibody positivity and the age of the patient. To explore the relationship between history of insulin therapy or diabetic ketoacidosis (DKA) at the onset of the disease with antibody positivity in a subsample. Material and Methods: Data was gathered from every sample processed for DM1 antibodies in our laboratory between January 2015 and September 2019. Medical records from 84 patients who tested positive for at least one antibody were revised to study the relationship between insulin therapy or DKA at the onset of the disease with antibody positivity. Results: Forty percent of DM1 antibody tests were positive. Among positive tests, 1, 2, 3 or 4 DM1 antibodies were detected in $48 \%, 33 \%, 17 \%$ and $3 \%$ of cases, respectively. The likelihood of testing positive was inversely related with age for ICA, GAD, IA-2, $\mathrm{ZnT8}$ and directly proportional for IAA $(\beta=-0,012 ;-0,013 ;-0,014 ;-0,009 ; 0,005$ respectively). An association between DKA at the onset of the disease and IA-2 positivity was observed (Odds ratio (OR) 5.38 95\% confidence intervals (CI) $1.79-16.16, P<0.01)$. No association was found between IAA positivity and history of insulin therapy (OR 2.25 95\% CI 0.63 - 7.90, P = 0.2403). Conclusions: The results obtained from this study represent a novel local profile of DM1 antibody data, highlighting a relationship between antibody positivity and age.

(Rev Med Chile 2021; 149: 330-338)

Key words: Antibodies; Diabetic Ketoacidosis; Diabetes Mellitus, Type 1; Insulin Antibodies; Immunoassay.
L a diabetes mellitus tipo 1 (DM1) es una enfermedad autoinmune crónica que ocudenada por factores ambientales ${ }^{1,2}$. La incidencia de DM1 en la infancia continúa incrementándose anualmente $3-4 \%$ a nivel mundial ${ }^{3,4}$. La insulitis $\mathrm{y}$ autoinmunidad mediada por linfocitos $\mathrm{T}$ son los mecanismos iniciales de destrucción de las células beta del páncreas ${ }^{2,5}$. Sin embargo, no todos los pacientes con DM1 poseen estas características fisiopatológicas; llevando a la clasificación de DMla (autoinmune) que corresponde a un 70-90\% de los casos y DM1b (idiopática) que representa al resto y cuya patogénesis específica aún no está clara ${ }^{6}$. 
En el contexto de DM1a, la detección de autoanticuerpos marcadores de DM1 se considera un fenómeno asociado a la insulitis, describiéndose la presencia de al menos 1 autoanticuerpo en $>95 \%$ de pacientes con DM1 al momento de detección de hiperglicemia ${ }^{2,7}$. Entre los autoanticuerpos asociados a DM1 se incluyen los antidecarboxilasa del ácido glutámico (GAD), antiinsulina (IAA), antitirosina fosfatasa IA-2 (IA-2), anticélula beta (ICA) y antiisoforma 8 del transportador de zinc (ZnT8). La seroconversión de autoanticuerpos suele producirse en forma individual, en un plazo de meses a años ${ }^{8}$. Aparte de su sensibilidad diagnóstica, los marcadores inmunológicos de diabetes han sido evaluados como predictores de historia natural de la enfermedad. Es así como algunos estudios sugieren que pacientes diabéticos con títulos elevados de GAD padecen una respuesta autoinmune grave y mayor riesgo de requerir insulinoterapia ${ }^{9,10}$. La presencia de mayor número de autoanticuerpos positivos se asociaría también con pérdida de función de células beta y progresión de enfermedad ${ }^{11}$. Por otro lado, la frecuencia de autoanticuerpos en DM1 varía con la edad de los pacientes y la ubicación geográfica, no existiendo datos descritos en Chile ${ }^{12}$.

La presente investigación corresponde a un perfil descriptivo de 5 autoanticuerpos asociados a DM1: GAD, IAA, IA-2 e ICA medidos entre enero 2015 y septiembre 2019, y ZnT8, incorporado a nuestro laboratorio desde noviembre de 2018. El objetivo principal de este estudio es determinar la frecuencia y combinaciones de positividad de los marcadores inmunológicos de DM1 al momento de su solicitud, y la relación de positividad de cada anticuerpo con la edad. Además, de un total de 1.683 pacientes positivos para los marcadores, se estudió una submuestra de 84 pacientes para los cuales se tuvo acceso a su historia clínica. En estos, se relacionó el antecedente de insulinoterapia con la positividad del anticuerpo IAA y se evaluó la relación de cetoacidosis diabética (CAD) con la positividad de los diferentes autoanticuerpos.

\section{Material y Método}

\section{Diseño general del estudio}

Este estudio de tipo transversal está compuesto de dos fases: análisis de resultados históricos de laboratorio y análisis de datos clínicos. Para la primera fase, se recopilaron los resultados de todas las muestras procesadas para marcadores inmunológicos de diabetes en el Laboratorio de Bioquímica del Centro Médico San Joaquín, Red de Salud UC-CHRISTUS, entre enero de 2015 y septiembre de 2019, lo que se consideró como el universo de estudio $(\mathrm{n}=4.164)$. Las determinaciones de los distintos marcadores de diabetes se realizaron mediante las siguientes metodologías:

Enzyme-Linked ImmunoSorbent Assay (ELISA) GAD (punto de corte $\geq 10 \mathrm{UI} / \mathrm{mL}$ ), IA-2 (punto de corte $\geq 10 \mathrm{UI} / \mathrm{mL}$ ) (EUROIMMUN, Alemania); $\mathrm{ZnT} 8$ (punto de corte $\geq 15 \mathrm{U} / \mathrm{mL}$ ) (ElisaRSR ${ }^{\mathrm{TM}}$, RSR Limited, UK) e IAA, (Isletest ${ }^{\mathrm{TM}}$-IAA, BIOMERICA, USA); Inmunofluorescencia indirecta ICA, (positivo en dilución 1:10) (EUROIMMUN, Alemania). Los calibradores utilizados en los ensayos de ELISA para GAD e IA-2 se encuentran estandarizados contra el material de referencia código 97/550 del National Institute for Biological Standards and Control (NIBSC) de la Organización Mundial de la Salud (OMS) incluidos desde el año 2000 en el Diabetes Antibody Standardization Program (DASP) para asegurar la calidad de los méto$\operatorname{dos}^{13,14}$. Se calcularon frecuencias y proporciones de positividad de los marcadores de diabetes, de forma aislada y en combinaciones.

En la segunda fase se revisó la historia clínica de 84 pacientes con al menos un marcador positivo atendidos en la Red de Salud UC-CHRISTUS, con el propósito de: (a) investigar el antecedente de exposición a insulina previa a la determinación de los marcadores y la positividad de IAA; (b) evaluar si el debut de DM1 con CAD se correlaciona con el número y tipo de marcadores positivos.

La relación edad/frecuencia de positividad se estableció a través de la regresión Poisson. Para estudiar la magnitud de asociación entre la positividad del marcador IAA y el antecedente de insulinoterapia, así como entre el debut con CAD y la positividad de marcadores se calculó una razón de probabilidades (Odds Ratio, OR). Se realizó la prueba exacta de Fisher para establecer significancia. Los gráficos y cálculos se realizaron con apoyo del software GraphPad Prism 8.3 ${ }^{\oplus}$.

El protocolo de recolección de datos y revisión de fichas clínicas fue aprobado por el Comité de Ética de la Facultad de Medicina de la Pontificia Universidad Católica de Chile. 


\section{Resultados}

\section{Frecuencia y combinaciones de positividad de los marcadores inmunológicos de DM1}

La edad promedio del universo de estudio $(\mathrm{n}=4164)$ fue de $34,32 \pm 17,87$ años, siendo $2113 / 4164$ individuos de sexo masculino $(50,74 \%)$. De los perfiles solicitados, $1.683 / 4.164$ resultaron positivos $(40,42 \%)$. En $47,83 \%$, $32,50 \%, 17,17 \%$ y $2,50 \%$ de los casos positivos se detectaron 1, 2, 3 o 4 marcadores, respectivamente. Los 4 marcadores fueron detectados simultáneamente en pacientes con edad promedio de 23,44 $\pm 16,97$ años (rango de edad de 0 a 73 años). El marcador de mayor prevalencia fue GAD, presente en $73,74 \%$ de los perfiles positivos, siendo, además, el único marcador detectado en $24 \%$ de estos (Figura 1). La segunda frecuencia de presentación individual fue IAA, con 16,70\%, aumentando a $30,96 \%$ al incluir combinaciones. De las combinaciones de anticuerpos, las más frecuentes correspondieron a GAD/IA-2 y GAD/ IA-2/ICA, con $22,58 \%$ y $11,94 \%$ de los casos, respectivamente.

Para 765 pacientes se estudió, además, la frecuencia de marcadores tras agregar ZnT8 a la batería de prestaciones desde noviembre de 2018. Se detectaron 339/765 pacientes con al menos un marcador positivo $(44,31 \%), 178 / 339$ positivos para ZnT8 $(52,50 \%)$ y $18 / 339$ pacientes positivos solo para ZnT8 (5,31\%). Se identificaron 15/339 casos con los 5 marcadores positivos (4,42\%).

\section{Relación de positividad de los marcadores inmunológicos de DM1 con la edad al momento de detección}

La probabilidad de positividad al momento de determinación fue inversa a la edad para ICA, GAD, IA-2 y ZnT8, reflejado por valores $\beta$ negati- vos de la regresión Poisson $(-0,012 ;-0,013 ;-0,014$; $-0,009$, respectivamente) (Figura 2$)$. Caso opuesto fue el de IAA, con un $\beta$ positivo $(0,005)$. Además, la distribución de edades con mayor probabilidad de positividad a la determinación fue significativamente mayor $(p \leq 0,0001)$ en el grupo de IAA en comparación a los demás marcadores. Así mismo, utilizando un punto de corte de 15 años, se calculó una asociación entre la edad mayor a 15 años y la positividad para los distintos marcadores de forma aislada o en combinación, encontrándose una relación en este grupo etario para IAA positivo de forma aislada (OR 7,33 IC $_{95 \%} 4,49-11,97$; $\mathrm{p}<0,0001$ ), IAA positivo o más marcadores (OR $3,46, \mathrm{IC}_{95 \%} 2,62-4,57$; $\left.\mathrm{p}<0,0001\right)$ y GAD positivo de forma aislada (OR 1,48, IC $_{95 \%}$ 1,14-1,92; $\mathrm{p}=0,0031$ ), caso contrario donde el resto de los marcadores y combinaciones se relacionan con una edad menor o igual a 15 años (Figura 3).

\section{Análisis de los antecedentes en ficha clínica para una submuestra de 84 pacientes}

La edad promedio de la muestra estudiada ( $\mathrm{n}=84$ ) fue de 23,44 $\pm 16,9$ años, correspondiendo $54,76 \%$ a sexo masculino. Cuarenta y dos de 84 pacientes $(50 \%)$ tenían el antecedente de uso previo de insulina a la determinación de marcadores y 33/84 (39,2\%) debutaron con CAD. La edad promedio del grupo de debut con CAD fue $14,64 \pm 13,61$ años y sin CAD 29,14 $\pm 16,59$ años.

Los marcadores, de forma aislada o combinada, presentaron la siguiente tasa de positividad: ICA $18 / 84$ (21,42\%); IAA $12 / 84$ (14,28\%); GAD 68/84 (80,95\%); IA-2 54/84 (64,28\%). Treinta y cinco de 84 pacientes $(41,66 \%)$ presentaron uno, $32 / 84(38,09 \%)$ dos, $15 / 84(17,85 \%)$ tres y $2 / 84$ $(2,38 \%)$ cuatro marcadores positivos de manera concomitante. En el caso de ZnT8, solo 25/84 pacientes de la muestra tenían realizada esta prue-

\begin{tabular}{|c|r|r|r|r|r|r|r|r|r|r|r|r|r|r|r|r|r|r|}
\hline \multicolumn{10}{|c|}{ Total } \\
\hline $\mathbf{n}$ & 404 & 380 & 281 & 201 & 107 & 84 & 73 & 45 & 42 & 20 & 13 & 12 & 12 & 6 & 3 & 1683 \\
\hline$\%$ & 24 & 22,58 & 16,7 & 11,94 & 6,36 & 4,99 & 4,34 & 2,67 & 2,5 & 1,19 & 0,77 & 0,71 & 0,71 & 0,36 & 0,18 & 100 \\
\hline GAD & + & & & & & & & & & & & & & & & \\
\hline IA2 & & & & + & & & & & & & & & & \\
\hline IAA & & & + & & & & & & & & & & & & \\
\hline ICA & & & & & & & & & & + & & & & & \\
\hline
\end{tabular}

Figura 1. Frecuencia y combinaciones de positividad de los marcadores de DM1 al momento de su solicitud. "+" identifica la detección de solo un tipo de anticuerpo. GAD: antidecarboxilasa del ácido glutámico, IAA: antiinsulina, IA-2: antitirosina fosfatasa, ICA: anticélula beta, ZnT8: antiisoforma 8 del transportador de zinc. 


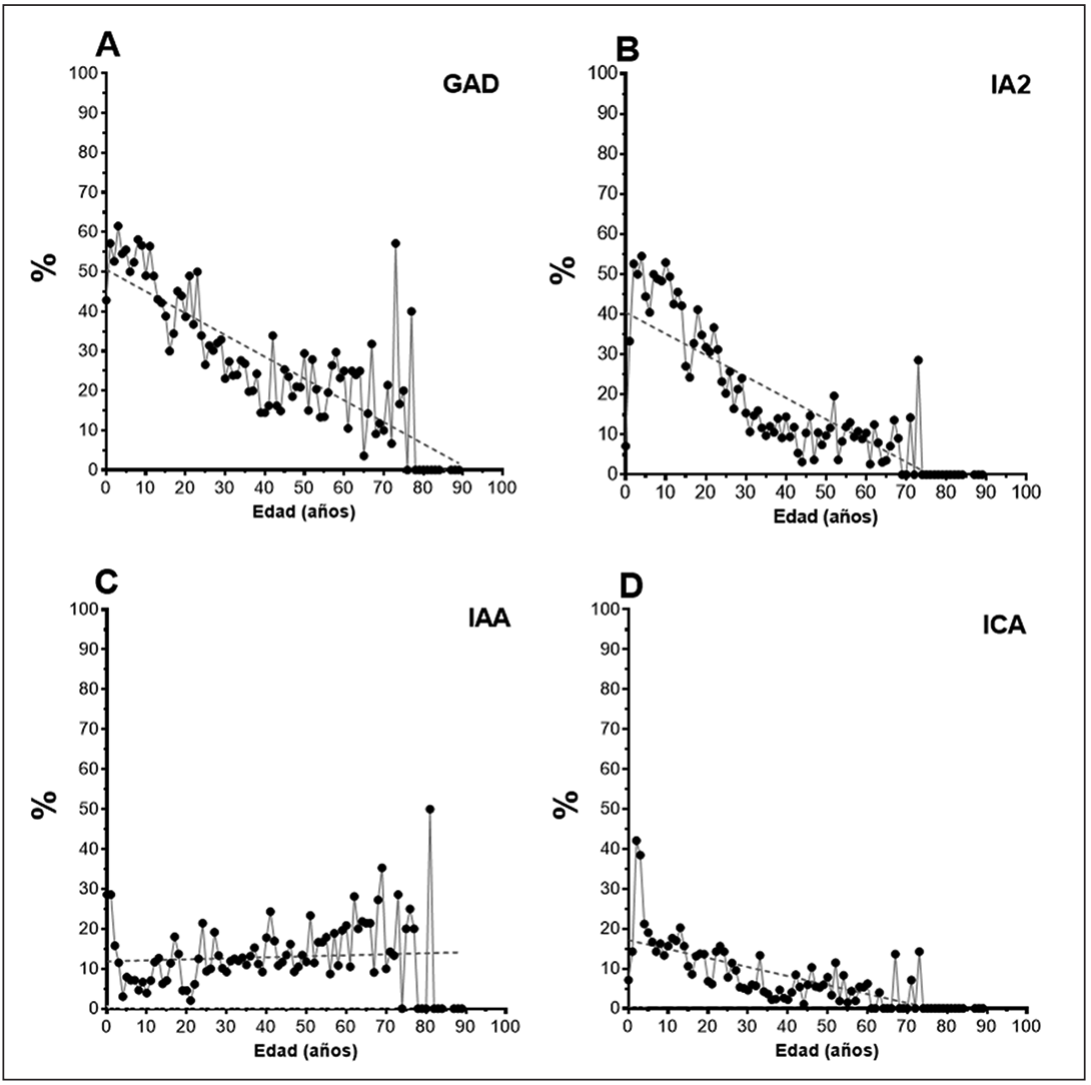

Figura 2. Relación entre tasa de positividad (determinaciones positivas/ total determinaciones $x$ 100) y edad en el universo de estudio. GAD: antidecarboxilasa del ácido glutámico, IAA: antiinsulina, IA-2: antitirosina fosfatasa, ICA: anticélula beta.

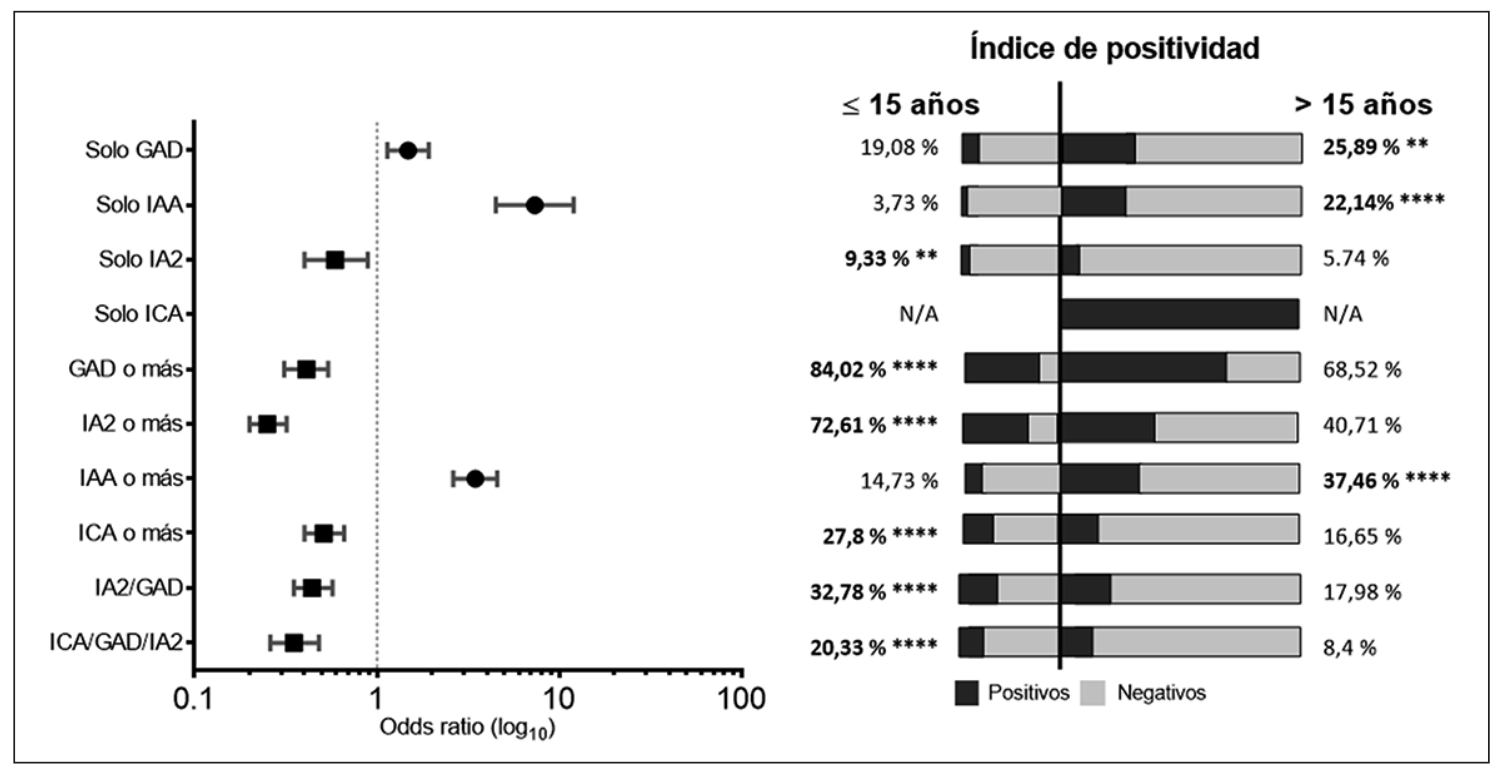

Figura 3. Relación entre positividad de marcadores (de forma aislada o en combinación) y edad > 15 años en el universo de estudio. GAD: antidecarboxilasa del ácido glutámico, IAA: antiinsulina, IA-2: antitirosina fosfatasa, ICA: anticélula beta. $* * * * p<0,0001 ; * * * p<0,001 ;{ }^{* *} p<0,005 ;{ }^{*} p<0,05 ;$ ns $p>0,05$. 


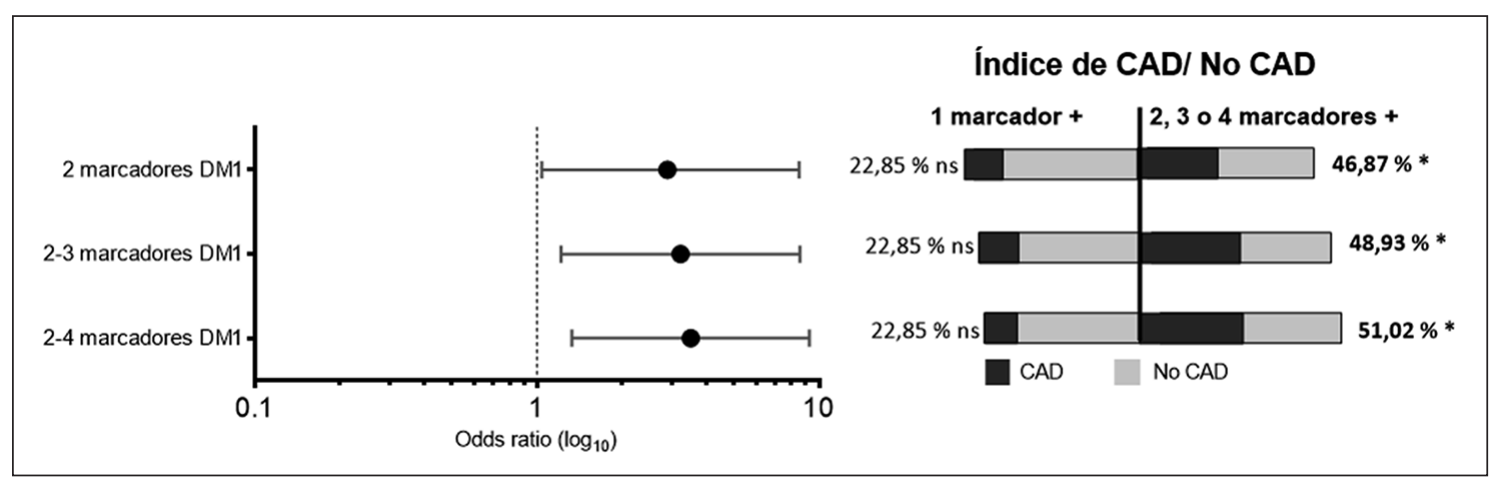

Figura 4. Relación entre debut con cetoacidosis diabética y número de marcadores positivos. DM1: diabetes mellitus tipo 1 , CAD: cetoacidosis diabética. ${ }^{* * * *} p<0,0001 ;{ }^{* * *} p<0,001 ;{ }^{* *} p<0,005 ;{ }^{*} p<0,05$; ns $p>0,05$.

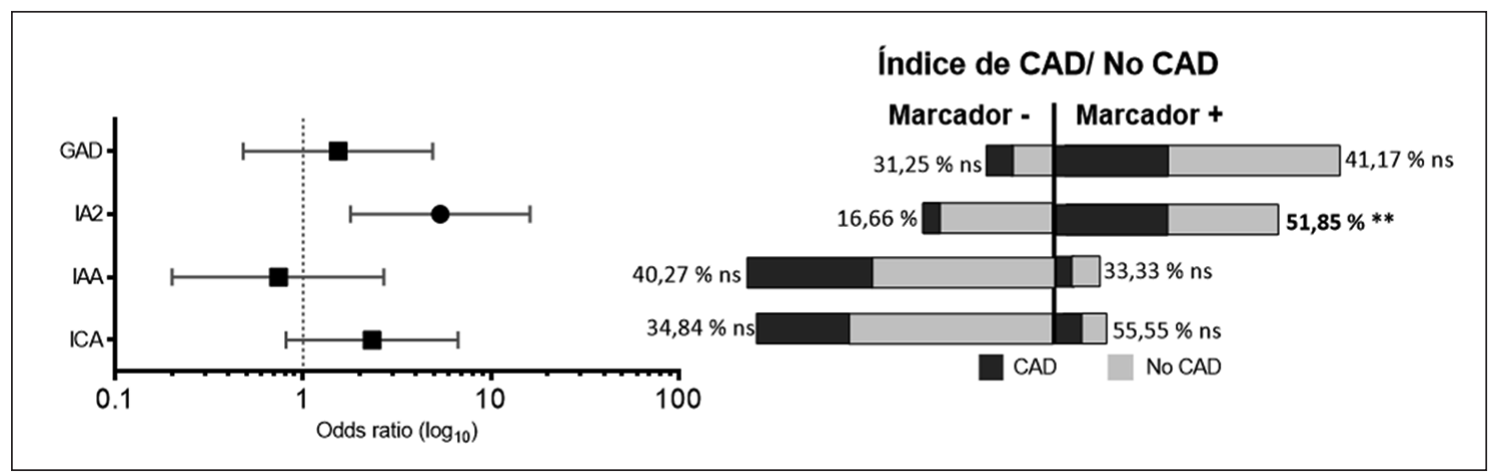

Figura 5. Relación entre debut con cetoacidosis diabética y positividad (solo o en combinación) de cada marcador. GAD: antidecarboxilasa del ácido glutámico, IAA: antiinsulina, IA-2: antitirosina fosfatasa, ICA: anticélula beta, CAD: cetoacidosis diabética. ${ }^{* * *} \mathrm{p}<0,0001 ;{ }^{* * *} \mathrm{p}<0,001 ;{ }^{* *} \mathrm{p}<0,005 ;{ }^{*} \mathrm{p}<0,05 ;$ ns $\left.\mathrm{p}>0,05\right)$.

ba, de las cuales 22/25 fueron positivas de forma aislada o combinada $(78,57 \%)$.

Se determinó una asociación entre debut con CAD y tener dos marcadores DM1 positivos de manera concomitante, resultando un OR de 2,9 $\left(\mathrm{IC}_{95 \%} 1,04-8,52 ; \mathrm{p}=0,044\right)$ en comparación a tener solo un marcador positivo, aumentando a 3,23 $\left(\mathrm{IC}_{95 \%} 1,22-8,57 ; \mathrm{p}=0,0215\right)$ con $2-3$ marcadores positivos y $3,51\left(\mathrm{IC}_{95 \%} 1,33-9,25, \mathrm{p}=0,0126\right)$ con 2-4 marcadores positivos (Figura 4).

En el caso de la asociación entre la positividad de cada marcador y el debut con CAD (Figura 5), se encontró una relación con IA-2, obteniéndose un OR de 5,38 $\left(\mathrm{IC}_{95 \%} 1,79-16,16 ; \mathrm{p}=0,0022\right)$ no siendo así para los demás marcadores: GAD, OR $1,54\left(\mathrm{IC}_{95 \%} 0,48-4,9 ; \mathrm{p}=0,464\right) ; \mathrm{IAA}, \mathrm{OR}\left(\mathrm{IC}_{95 \%}\right.$ $0,20-2,69 ; \mathrm{p}=0,756)$; ICA, OR 2,33 ( $\mathrm{IC}_{95 \%} 0,81-$ $6,70 ; \mathrm{p}=0,17)$.
No se observó relación entre la positividad del marcador IAA y el antecedente de uso previo de insulina (OR 2,25 $\left.\mathrm{IC}_{95 \%} 0,63-7,90, \mathrm{p}=0,2403\right)$.

\section{Discusión}

Estudios epidemiológicos realizados en Chile han evidenciado un incremento en la incidencia de DM1 en los últimos años ${ }^{15}$. En este contexto, el estudio de autoanticuerpos es un elemento de apoyo diagnóstico, clasificación y predicción de enfermedad.

El alto porcentaje de positividad de los perfiles evaluados (40,42\%) sugiere que la solicitud de estos marcadores se realiza con elevada sospecha clínica. La mitad de los perfiles presentaba más de 1 anticuerpo positivo, siendo conocido que 
el número de marcadores detectados es el mayor predictor de progresión a DM1 ${ }^{16,17}$. En pacientes con diagnóstico reciente de DM1, se ha visto que hasta $98 \%$ presenta al menos 1 anticuerpo y más del 75\% al menos 2 anticuerpos, siendo GAD el más frecuente $e^{18,19}$. Los autoanticuerpos tienden a positivar secuencialmente en vez de simultáneamente, primero para GAD o IAA y luego IA-2. Entre aquellos que expresan más de 1 marcador, $2 / 3$ expresan GAD primero y $1 / 3$ seroconvierte primero para IAA ${ }^{20}$. Concordantemente, en nuestro estudio, GAD es el anticuerpo de mayor frecuencia y en segundo lugar IAA.

Inicialmente se describió que la administración de insulina exógena era capaz de inducir la producción de IAA $^{21}$. Sin embargo, estudios ya han demostrado la detección de IAA en pacientes con DM1 previo a la insulinoterapia e incluso años antes del diagnóstico de diabetes, siendo concordante con la ausencia de relación entre el uso de insulina exógena y la positividad de IAA descrita en nuestros resultados ${ }^{22,23}$. IAA se ha detectado en hasta 70\% de pacientes con sospecha de DM1, considerándose mejor predictor de enfermedad en niños que en adultos ${ }^{24}$. Estudios prospectivos basados en seguimiento desde el nacimiento (DiPP, DAISY, BABYDIAB y TEDDY) sugieren que IAA es el primer autoanticuerpo detectado antes de los 4 años, con picos descritos incluso alrededor del año de edad ${ }^{8,23}$.

Debido a la variación interlaboratorio inicial, el Diabetes Autoantibody Standardization Program (DASP) ha liderado talleres de estandarización metodológica. Los ELISAs para GAD e IA-2 han alcanzado rendimiento comparable a los radioinmunoensayos $^{14}$. Sin embargo, el ELISA para IAA persiste con baja sensibilidad ${ }^{25}$, sin alcanzar estándares DASP aceptables ${ }^{26}$. Esto explicaría la menor detección de IAA en nuestra población comparada con cohortes previas estudiadas con radioinmunoensayos. Entonces, si bien en la literatura GAD/ IAA es la combinación de autoanticuerpos más sensible para el diagnóstico de DM1 autoinmune en adultos ${ }^{19}$, su detección en solo $5 \%$ de nuestros pacientes con marcadores positivos puede ser efecto de una baja sensibilidad analítica del ELISA IAA. Por este mismo motivo, nuestra población presentaría un mayor porcentaje de pacientes con GAD aislado o GAD/IA-2.

Nuestros datos reflejan una relación inversa entre la positividad de GAD, ICA e IA-2 y la edad, es decir, a menor edad mayor fue el porcentaje de positividad para estos anticuerpos y el caso opuesto se observó para IAA, donde el porcentaje de positividad fue mayor a mayor edad. Dado el corte transversal de nuestro estudio, estos resultados no pueden ser comparados con estudios prospectivos previos. Además, encontramos 4 marcadores de forma simultánea en pacientes con un rango de edad de 1 a 73 años, hallazgos descritos en estudios de adultos mayores ${ }^{27}$. Se debe recordar que la detección de autoanticuerpos no equivale necesariamente al diagnóstico de DM1, ya que en grandes cohortes, estos marcadores han sido detectados en hasta 9,7\% de sujetos inicialmente clasificados como DM $2^{28}$ considerados por algunos grupos como latent autoimmune diabetes in adult (LADA) ${ }^{29}$.

El debut de DM1 con CAD es más común en población pediátrica, especialmente en el rango de 0 a 4 años ${ }^{30-32}$. En la muestra estudiada, la edad del grupo con debut CAD es significativamente menor comparado con aquellos sin CAD, siendo concordante con la literatura ${ }^{33}$, si bien existen otras variables de carácter no biológico que están relacionadas con el debut con $\mathrm{CAD}^{30-32}$.

Más del 60\% de los pacientes adultos con DM con tendencia a CAD carecen de evidencia de autoinmunidad ${ }^{34,35}$. Es conocido que los sueros de pacientes con anti-IA-2 (IA-2 e IA-2 $\beta$ ) reaccionan con su dominio intracelular ${ }^{36}$ y los constructos usados en los ensayos de las pruebas de rutinas solo incluyen este dominio y no el extracelular $(\mathrm{IA}-2 \mathrm{EC})^{37}$. Ha sido reportado que el $1 \%$ de los pacientes con DM1 son positivos solo para el anticuerpo IA-2EC y 4,7\% con DM2 $2^{37,38}$. En una cohorte de pacientes $(n=288)$ con tendencia a CAD negativos para los marcadores, se identificaron 3,5\% pacientes positivos para el IA-2-EC ${ }^{39}$. Aunque la CAD fue la primera manifestación de DM para algunos pacientes, muchos habían tenido diabetes durante tiempos variables antes de su primer episodio de CAD, la medición de IA-2-EC podría proporcionar datos útiles para clasificar y manejar pacientes con tendencia a CAD y su presencia podría predecir el requerimiento de insulina a largo plazo.

$\mathrm{Al}$ agregar ZnT8 a la batería solicitada, 52,50\% de los perfiles positivos presentaba este marcador. Para 5,31\% de los casos, ZnT8 fue el único marcador detectado, aumentando con estos casos la sensibilidad global de la batería. Además, convirtió 
a pacientes con un único marcador en "múltiple positivo", lo cual se asocia en la literatura a un aumento en el riesgo de $\mathrm{DM} 1^{40}$.

Debido al corte transversal de este estudio, el sesgo de memoria podría explicar la falta de asociación entre el uso de insulina e IAA, ya que no todos los pacientes podrían recordar su uso aislado, en conjunto con limitación metodológica (la falta de estándares DASP aceptables) para su determinación ${ }^{26}$. A esto se suma la falta de antecedentes (diagnóstico, indicación de solicitud de los marcadores y patología asociada) del universo analizado. En cuanto al poder estadístico, es necesario destacar que la muestra clínica ( 84 pacientes) fue elegida por conveniencia, sujeta a la disponibilidad de fichas clínicas, restándole representatividad respecto al universo de estudio.

A pesar de sus limitaciones, este estudio corresponde al primer análisis de marcadores inmunológicos de DM1 en Chile, e invita a la comunidad científica a continuar explorando asociaciones entre la positividad de estos marcadores y las características clínicas de personas con DM1.

\section{Referencias}

1. Schwartz SS, Epstein S, Corkey BE, Grant SFA, Gavin JR, Aguilar RB. The time is right for a new classification system for diabetes: Rationale and implications of the $\beta$-cell-centric classification schema. Diabetes Care. American Diabetes Association Inc. 2016; 179-86. [Consultado el 19 de noviembre de 2019].

2. Paschou SA, Papadopoulou-Marketou N, Chrousos GP, Kanaka-Gantenbein C. On type 1 diabetes mellitus pathogenesis. Endocrine Connections. BioScientifica Ltd. 2018; R38-46. [Consultado el 19 de noviembre de 2019].

3. Patterson CC, Dahlquist GG, Gyürüs E, Green A, Soltész $\mathrm{G}$, Schober E, et al. Incidence trends for childhood type 1 diabetes in Europe during 1989-2003 and predicted new cases 2005-20: a multicentre prospective registration study. Lancet. 2009; 373 (9680): 2027-33. [Consultado el 19 de noviembre de 2019].

4. Mayer-Davis EJ, Lawrence JM, Dabelea D, Divers J, Isom S, Dolan L, et al. Incidence trends of type 1 and type 2 diabetes among youths, 2002-2012. N Engl J Med. 2017; 376 (15): 1419-29. [Consultado el 19 de noviembre de 2019].

5. Morgan NG. Bringing the human pancreas into focus: new paradigms for the understanding of Type 1 diabe- tes. Diabetic Medicine. Blackwell Publishing Ltd; 2017; 879-86. [Consultado el 20 de noviembre de 2019].

6. Atkinson MA, Eisenbarth GS, Michels AW. Type 1 diabetes. The Lancet. Lancet Publishing Group; 2014; 383 (9911): 69-82. [Consultado el 12 de noviembre de 2019]

7. Calderon B, Sacks DB. Islet autoantibodies and type 1 diabetes: Does the evidence support screening? Clinical Chemistry. American Association for Clinical Chemistry Inc. 2014; 60: 438-40. [Consultado el 10 de octubre de 2019].

8. Bosi E, Boulware DC, Becker DJ, Buckner JH, Geyer S, Gottlieb PA, et al. Impact of age and antibody type on progression from single to multiple autoantibodies in type 1 diabetes relatives. J Clin Endocrinol Metab. 2017; 102 (8): 2881-6. [Consultado el 20 de noviembre de 2019].

9. Yasui J, Kawasaki E, Tanaka S, Awata T, Ikegami H, Imagawa A, et al. Clinical and Genetic Characteristics of Non-Insulin-Requiring Glutamic Acid Decarboxylase (GAD) Autoantibody-Positive Diabetes: A Nationwide Survey in Japan. Pietropaolo M, editor. PLoS One. 2016; 11 (5): e0155643. Disponible en: https://dx.plos. org/10.1371/journal.pone.0155643[Consultado el 3 de enero de 2020].

10. Buzzetti R, Di Pietro S, Giaccari A, Petrone A, Locatelli $\mathrm{M}$, Suraci C, et al. High titer of autoantibodies to GAD identifies a specific phenotype of adult-onset autoimmune diabetes. Diabetes Care. 2007; 30 (4): 932-8. [Consultado el 19 de noviembre de 2019].

11. Ziegler AG, Rewers M, Simell O, Simell T, Lempainen J, Steck A, et al. Seroconversion to multiple islet autoantibodies and risk of progression to diabetes in children. JAMA - J Am Med Assoc. 2013; 309 (23): 2473-9. [Consultado el 18 de noviembre de 2019].

12. Report of the expert committee on the diagnosis and classification of diabetes mellitus. Diabetes Care. 1997 Jul; 20 (7): 1183-97. [Consultado el 19 de noviembre de 2019].

13. Mire-Sluis AR, Gaines Das R, Lernmark $\AA$. The World Health Organization international collaborative study for islet cell antibodies. Diabetologia. 2000; 43 (10): 1282-92 [Consultado el 21 de noviembre de 2019].

14. Törn C, Mueller PW, Schlosser M, Bonifacio E, Bingley PJ. Diabetes Antibody Standardization Program: Evaluation of assays for autoantibodies to glutamic acid decarboxylase and islet antigen-2. Diabetologia. 2008; 51 (5): 846-52 [Consultado el 21 de noviembre de 2019].

15. Díaz N, Méndez MA, Pérez-Bravo F, Carrasco E, Santos JL. Incidence rate of type 1 diabetes in Santiago (Chile) by HLA-DQA1 and DQB1 genotypes. Eur J Epidemiol. 2002; 18 (8): 787-92. Disponible en: http://link.springer. 
com/10.1023/A:1025345804789 [Consultado el 23 de noviembre de 2019].

16. Incani M, Serafini C, Satta C, Perra L, Scano F, Frongia $\mathrm{P}$, et al. High prevalence of diabetes-specific autoimmunity in first-degree relatives of Sardinian patients with type 1 diabetes. Diabetes Metab Res Rev. 2017; 33 (3): e2864. Disponible en: http://www.ncbi.nlm.nih.gov/ pubmed/27726307 [Consultado el 10 de diciembre de 2019].

17. Orban T, Sosenko JM, Cuthbertson D, Krischer JP, Skyler JS, Jackson R, et al. Pancreatic Islet Autoantibodies as Predictors of Type 1 Diabetes in the Diabetes Prevention Trial-Type 1. Diabetes Care. 2009; 32 (12): 2269-74. Disponible en: http://www.ncbi.nlm.nih.gov/ pubmed/19741189 [Consultado el 10 de diciembre de 2019].

18. Verge CF, Gianani R, Kawasaki E, Yu L, Pietropaolo $\mathrm{M}$, Chase HP, et al. Number of autoantibodies (against insulin, GAD or ICA512/IA2) rather than particular autoantibody specificities determines risk of type I diabetes. Journal of Autoimmunity. Academic Press 1996; 379-83. [Consultado el 10 de diciembre de 2019].

19. Betterle C, Lazzarotto F, Fusari A, Zanchetta R, Benedini S, Pedini B, et al. Pancreatic autoantibodies in Italian patients with newly diagnosed type 1 diabetes mellitus over the age of 20 years. Acta Diabetol. 2006; 43 (3): 79-83. Disponible en: http://www.ncbi.nlm.nih.gov/ pubmed/17143785 [Consultado el 10 de diciembre de 2019].

20. Yu L, Rewers M, Gianani R, Kawasaki E, Zhang Y, Verge $\mathrm{C}$, et al. Antiislet autoantibodies usually develop sequentially rather than simultaneously. J Clin Endocrinol Metab. 1996; 81 (12): 4264-7. Disponible en: https:// www.ncbi.nlm.nih.gov/pubmed/8954025 [Consultado el 3 de enero de 2020].

21. Yalow RS, Berson SA. Assay of plasma insulin in human subjects by immunological methods. Nature. 1959; 184 (4699): 1648-9. [Consultado el 2 de enero de 2020].

22. Palmer JP, Asplin CM, Clemons P, Lyen K, Tatpati O, Raghu PK, et al. Insulin antibodies in insulin-dependent diabetics before insulin treatment. Science. 1983; 222 (4630): 1337-9. [Consultado el 2 de enero de 2020].

23. Rewers M, Hyöty H, Lernmark $\AA$, Hagopian W, She JX, Schatz D, et al. The Environmental Determinants of Diabetes in the Young (TEDDY) Study: 2018 Update. Curr Diab Rep. 2018; 18 (12): 136. Disponible en: https://www.ncbi.nlm.nih.gov/pmc/articles/PMC6415767/ [Consultado el 2 de enero de 2020].

24. Zimmet PZ, Elliott RB, Mackay IR, Tuomi T, Rowley MJ, Pilcher CC, et al. Autoantibodies to Glutamic Acid Decarboxylase and Insulin in Islet Cell Antibody Positi- ve Presymptomatic Type 1 Diabetes Mellitus: Frequency and Segregation by Age and Gender. Diabet Med. 1994; 11 (9): 866-71. Disponible en: http://www.ncbi.nlm.nih. gov/pubmed/7705024 [Consultado el 10 de diciembre de 2019].

25. Greenbaum CJ, Palmer JP, Kuglin B, Kolb H, Arnaiz-Villena A, Beaufort CDD, et al. Insulin autoantibodies measured by radioimmunoassay methodology are more related to insulin-dependent diabetes mellitus than those measured by enzyme-linked immunosorbent assay: Results of the Fourth International Workshop on the Standardization of Insulin Autoantibody Measurement. J Clin Endocrinol Metab. 1992; 74 (5): 1040-4. Disponible en: http://www.ncbi.nlm.nih.gov/pubmed/7705024 [Consultado el 10 de diciembre de 2019].

26. Bingley PJ, Bonifacio E, Mueller PW. Diabetes antibody standardization program: First assay proficiency evaluation. Diabetes. 2003; 52 (5): 1128-36. [Consultado el 10 de diciembre de 2019].

27. Thunander M, Petersson C, Jonzon K, Fornander J, Ossiansson B, Torn C, et al. Incidence of type 1 and type 2 diabetes in adults and children in Kronoberg, Sweden. Diabetes Res Clin Pract. 2008; 82 (2): 247-55. Disponible en: http://www.ncbi.nlm.nih.gov/pubmed/18804305 [Consultado el 10 de diciembre de 2019].

28. Pozzilli P, Pieralice S. Latent autoimmune diabetes in adults: Current status and new horizons. Endocrinol Metab (Seoul). 2018; 33 (2): 147-59. Disponible en: https://www.ncbi.nlm.nih.gov/pmc/articles/PMC6021307/ [Consultado el 19 de diciembre de 2019].

29. Tuomi T, Groop LC, Zimmet PZ, Rowley MJ, Knowles W, Mackay IR. Antibodies to glutamic acid decarboxylase reveal latent autoimmune diabetes mellitus in adults with a non-insulin-dependent onset of disease. Diabetes. 1993; 42 (2): 359-62. Disponible en: https://diabetes. diabetesjournals.org/content/42/2/359.full-text.pdf [Consultado el 19 de diciembre de 2019].

30. Alonso GT, Coakley A, Pyle L, Manseau K, Thomas S, Rewers A. Diabetic Ketoacidosis at Diagnosis of Type 1 Diabetes in Colorado Children, 2010-2017. Diabetes Care. 2019;dc190428. Disponible en: https://care.diabetesjournals.org/content/43/1/117.long [Consultado el 19 de diciembre de 2019].

31. Jefferies C, Cutfield SW, Derraik JGB, Bhagvandas J, Albert BB, Hofman PL, et al. 15-year incidence of diabetic ketoacidosis at onset of type 1 diabetes in children from a regional setting (Auckland, New Zealand). Sci Rep. 2015; 5: 10358. Disponible en: https://www.ncbi.nlm. nih.gov/pmc/articles/PMC4650806/ [Consultado el 2 de enero de 2020].

32. Silverstein J, Klingensmith G, Copeland K, Plotnick L, 
Kaufman F, Laffel L, et al. Care of children and adolescents with type 1 diabetes: A statement of the American Diabetes Association. Diabetes Care. 200528 (1): 186212. Disponible en: https://care.diabetesjournals.org/ content/28/1/186.long [Consultado el 3 de enero de 2020].

33. Eyal O, Oren A, Almasi-Wolker D, Tenenbaum-Rakover Y, Rachmiel M, Weintrob N. Ketoacidosis in newly diagnosed type 1 diabetes in children and adolescents in israel: Prevalence and risk factors. Isr Med Assoc J. 2018; 20 (2): 100-3. Disponible en: https://www.ima.org.il/ FilesUploadPublic/IMAJ/0/272/136233.pdf [Consultado el 3 de enero de 2020].

34. Balasubramanyam A, Nalini R, Hampe CS, Maldonado M. Syndromes of ketosis-prone diabetes mellitus. Vol. 29, Endocrine Reviews. 2008; 29 (3): 292-302. Disponible en: https:/www.ncbi.nlm.nih.gov/pmc/articles/ PMC2528854/ [Consultado el 3 de enero de 2020].

35. Maldonado M, Hampe CS, Gaur LK, D’Amico S, Iyer D, Hammerle LP, et al. Ketosis-Prone Diabetes: Dissection of a Heterogeneous Syndrome Using an Immunogenetic and $\beta$-Cell Functional Classification, Prospective Analysis, and Clinical Outcomes. J Clin Endocrinol Metab. 2003; 88 (11): 5090-8. Disponible en: https://academic. oup.com/jcem/article/88/11/5090/2656268 [Consultado el 2 de enero de 2020].

36. Notkins AL, Zhang B, Matsumoto Y, Lan MS. Comparison of IA-2 with IA-2 $\beta$ and with Six Other Members of the Protein Tyrosine Phosphatase Family: Recognition of Antigenic Determinants by IDDM Sera. J Autoimmun. 11997; 10 (3): 245-50. Disponible en: https://www.
sciencedirect.com/science/article/pii/S0896841197901323 [Consultado el 26 de noviembre de 2019].

37. Morran MP, Casu A, Arena VC, Pietropaolo S, Zhang YJ, Satin LS, et al. Humoral autoimmunity against the extracellular domain of the neuroendocrine autoantigen IA-2 heightens the risk of type 1 diabetes. Endocrinology. 2010; 151 (6): 2528-37. Disponible en: https://www. ncbi.nlm.nih.gov/pmc/articles/PMC2875834/ [Consultado el 26 de noviembre de 2019].

38. Acevedo-Calado M, James EA, Morran MP, Pietropaolo SL, Ouyang Q, Arribas-Layton D, et al. Identification of Unique Antigenic Determinants in the Amino Terminus of IA-2 (ICA512) in Childhood and Adult Autoimmune Diabetes: New Biomarker Development. Diabetes Care. 2017; 40 (4): 561-8. Disponible en: http://www.ncbi. nlm.nih.gov/pubmed/28174261 [Consultado el 6 de diciembre de 2019].

39. Mulukutla SN, Acevedo-Calado M, Hampe CS, Pietropaolo M, Balasubramanyam A. Autoantibodies to the IA-2 extracellular domain refine the definition of "A+" subtypes of ketosis-prone diabetes. Diabetes Care. 2018; 1; 41 (12): 2637-40. Disponible en: https://www.ncbi. nlm.nih.gov/pmc/articles/PMC6245211/ [Consultado el 6 de diciembre de 2019].

40. Yu L, Boulware DC, Beam CA, Hutton JC, Wenzlau JM, Greenbaum CJ, et al. Zinc transporter-8 autoantibodies improve prediction of type 1 diabetes in relatives positive for the standard biochemical autoantibodies. Diabetes Care. 2012; 35 (6): 1213-8. Disponible en: https://www.ncbi.nlm.nih.gov/pmc/articles/PMC3357246/ [Consultado el 10 de diciembre de 2019]. 